\title{
Cardiovascular Therapeutics
}

\section{Diabetes and Cardiovascular Risk: Are Dipeptidyl Peptidase-4 Inhibitors Beneficial?}

\author{
Patricia A. Howard, PharmD, FCCP, BCPS (AQ CV)*
}

\begin{abstract}
Cardiovascular $(\mathrm{CV})$ disease is a major cause of morbidity and mortality in patients with diabetes. Whereas the link between glycemic control and reducing microvascular disease is firmly established, the evidence for macrovascular risk reduction remains unclear. Despite a host of available drugs for lowering serum glucose, none to date have been shown to substantially reduce CV risk and some have been associated with adverse effects. Recent trials have examined the CV effects of the dipeptidyl peptidase 4 (DPP-4) inhibitors or "gliptins."
\end{abstract}

$\mathrm{T}$ ype 2 diabetes affects an estimated 25.8 million individuals in the United States. ${ }^{1}$ Almost 2 million new cases were diagnosed in 2010 among adults 20 years of age and older. Data from the US Health and Nutritional Examination Survey (NHANES) indicate that the prevalence of diabetes increased dramatically from an estimated $6.2 \%$ for 1988-1994 to an estimated $9.9 \%$ for $2005-2010 .^{2}$

Diabetes is associated with accelerated atherosclerosis and is often considered to be a "coronary equivalent." The overall risks of cardiovascular disease (CVD) and stroke are 2 to 4 times higher in individuals with diabetes compared to nondiabetics. ${ }^{1}$ Despite the availability of numerous therapeutic agents with proven efficacy for lowering blood glucose, none have been shown to substantially reduce CV risk. Even more concerning is the fact that a number of these drugs have been associated with detrimental effects. For example, thiazolidinediones including rosiglitazone and pioglitazone, have been associated with an increased risk of heart failure resulting in a black box warning. ${ }^{3}$ Rosiglitazone has previously been associated with a potential increased risk of myocardial infarction and, although many of the restrictions on its use have now been removed, the US Food and Drug Administration (FDA) continues to monitor. ${ }^{4,5}$

Because CVD is a leading cause of death among diabetics, the development of additional antihyperglycemic drugs has provided an opportunity to determine the relative $\mathrm{CV}$ risk associated with these drugs. With this in mind, the FDA issued a 2008 guidance that manufacturers of new diabetes drugs should conduct studies to evaluate CV safety. ${ }^{6}$ The newest class of glucose-lowering drugs to undertake this assessment of CV risk is the dipeptidyl peptidase 4 (DPP-4) inhibitors. This article will discuss the findings from 2 recent clinical trials assessing the $\mathrm{CV}$ risk of these agents and the ongoing issues related to preventing macrovascular complications in patients with diabetes.

\section{DIPEPTIDYL PEPTIDASE 4 (DPP-4) INHIBITORS}

DPP-4 inhibitors, commonly referred to as "gliptins," block the degradation of incretin hormones that are involved in the regulation of glucose and insulin. ${ }^{7}$ The DPP-4 inhibitors were introduced in 2006. Due to their high cost, DPP-4 inhibitors are generally reserved for add-on therapy in patients who have not achieved adequate glycemic control with other drugs. Initial small studies suggested that DPP-4 inhibitors may have beneficial effects on the CV system. Increases in left ventricular contractility in patients with ischemic heart disease as well as reductions in blood pressure and postprandial lipid levels have been reported. ${ }^{7}$ In a subsequent meta-analysis of 70 trials involving almost 42,000 patients and 44 weeks of follow-up, DPP-4

"Professor and Vice Chair, Department of Pharmacy Practice, University of Kansas Medical Center, Mailstop 4047, 3901 Rainbow Boulevard, Kansas City, KS 66160; phone: 913-588-5391; fax: 913-588-2355; e-mail: phoward@kumc.edu 
inhibitors were associated with reductions in major adverse CV events (odds ratio [OR], 0.71; 95\% CI, $0.59-0.86 ; P<.001) .{ }^{8}$ A separate pooled analyses of data from the saxagliptin phase $2 b-3$ development program involving 4,607 patients showed lower rates of the combined endpoint of $\mathrm{CV}$ death/myocardial infarction/stroke (relative risk [RR], 0.43; 95\% CI, 0.23-0.80)..$^{9}$ In response to the FDA's guidance, 2 randomized placebo controlled trials evaluating CV risk were conducted, and the findings were reported at the European Society of Cardiology 2013 annual meeting. ${ }^{10,11}$

\section{CLINICAL TRIALS OF CARDIOVASCULAR RISK WITH DPP-4 INHIBITORS}

The first study, the Saxagliptin Assessment of Vascular Outcomes Recorded in Patients with Diabetes Mellitus-Thrombolysis in Myocardial Infarction (SAVOR-TIMI 53) trial, included 16,492 patients with type 2 diabetes, a glycated hemoglobin (A1C) of $6.5 \%$ to $12 \%$, and either a history of CVD or multiple risk factors. ${ }^{10}$ The study population had a mean age of 65 years and a median diabetes duration of 10 years. Saxagliptin was initiated at $5 \mathrm{mg}$ daily or $2.5 \mathrm{mg}$ for those with a glomerular filtration rate less than or equal to $50 \mathrm{~mL} / \mathrm{min}$. Physicians were allowed to adjust other medications, including antihyperglycemic agents, as needed. The primary endpoint was a composite of CV death, myocardial infarction, or ischemic stroke. After a median followup of 2.1 years, the primary endpoint occurred in $7.3 \%$ of saxagliptin versus $7.2 \%$ on placebo groups (hazard ratio [HR], 1.00; 95\% CI, 0.89-1.12; $P=.99$ for superiority; $P<.001$ for noninferiority). Unexpectedly, saxagliptin patients were found to be at increased risk for hospitalization due to heart failure $(3.5 \%$ vs $2.8 \% ; P=.007)$. The mean $\mathrm{A} 1 \mathrm{C}$ was $0.2 \%$ lower in the saxagliptin group compared to placebo $(P<.001)$ and hypoglycemia occurred more frequently with saxagliptin $(15.3 \%$ vs $13.4 \% ; P<.001)$.

The SAVOR-TIMI 53 investigators offered 3 possible explanations for the observed lack of CV benefit. First, the study duration may have been too short to positively impact CV outcomes. Second, the difference in A1Clevels was relatively small throughout the trial. The investigators attributed this to the fact that although baseline use of nonstudy glucoselowering drugs was similar between the groups, the use of insulin increased by approximately $5 \%$ in the placebo arm during the trial. Third, the overall event rates were relatively low, perhaps due to the widespread use of other cardioprotective drugs, thus making it more difficult to demonstrate significant differences. Reasons for the increase in heart failure hospitalizations were not clear.

The second study was the Examination of Cardiovascular Outcomes with Alogliptin vs Standard of Care in Patients with Type 2 Diabetes Mellitus and Acute Coronary Syndrome (EXAMINE) trial. ${ }^{11}$ This trial randomized 5,380 diabetic patients who were hospitalized for an acute coronary syndrome within the previous 15 to 90 days and had a baseline A1C of $6.5 \%$ to $11 \%$. Patients were initiated on alogliptin $25 \mathrm{mg}$ daily or placebo. Doses were modified for those with glomerular filtration rates below $60 \mathrm{~mL} / \mathrm{min}$. Additional antihyperglycemic and CV drugs were allowed as needed. Follow-up was conducted for a median of 18 months. The primary endpoint of death from CV causes, nonfatal myocardial infarction, or nonfatal stroke occurred in $11.3 \%$ of patients assigned to alogliptin compared to $11.8 \%$ of placebo patients $(P<.001$ for noninferiority and $P=.32$ for superiority). Alogliptin lowered A1C by an additional $0.3 \%$ and was not associated with increased hypoglycemia compared to placebo.

The EXAMINE investigators also commented that a longer duration trial may have been needed to demonstrate benefit. Reductions in A1C were small in this trial and similar to those seen in SAVORTIMI 53. Although the use of other antihyperglycemic drugs was matched at baseline, changes in the use of these agents by the end of the study were not reported.

\section{DISCUSSION}

Even though SAVOR-TIMI 53 and EXAMINE provide evidence that saxagliptin and alogliptin do not adversely affect CV endpoints such as myocardial infarction and stroke, the lack of any positive impact is disappointing. As is true for all studies, the design limitations must be taken into account when interpreting the findings. Along with the relatively small reductions in $\mathrm{A} 1 \mathrm{C}$ achieved in these trials, the lack of control over the use of nonstudy antihyperglycemic drugs is a potential confounder. Additionally, the trials were adequately powered and included over 20,000 patients, but the vast majority of the patients were White. Further studies are needed that include Black and Hispanic patients, 2 groups with even higher CV risks. The finding of increased heart failure hospitalizations with saxagliptin in SAVOR-TIMI 53 raises a cautionary flag that must be further examined. Unfortunately, heart failure hospitalizations have not been included as a predefined endpoint in any other 
gliptin studies. A recent meta-analysis pooled data from 84 studies to examine the relationship between DPP-4 inhibitors therapy and heart failure. ${ }^{12}$ The overall risk of acute heart failure was found to be higher with DPP-4 inhibitor therapy (OR, 1.19; 95\% CI, 1.03-1.37; $P=.015)$. The increased risk appeared to be a class effect as no differences were seen among the various DPP-4 inhibitors included in the analysis. In response to this concern, the FDA is currently conducting an analysis of the risk of heart failure associated with saxagliptin. ${ }^{13}$ Finally, although these 2 clinical trials were specifically designed to address the issue of CV risk, it is disappointing that microvascular outcomes were not included in the endpoints. Given the relatively small reductions in blood glucose levels achieved in both trials, one cannot assume that benefits would have occurred.

\section{Glycemic Control as a Surrogate for Vascular Disease}

Treatment goals for patients with diabetes focus on controlling blood glucose levels, which will in turn reduce target organ damage. Diabetes is associated with microvascular damage in the eyes, kidneys, and nerves as well as macrovascular damage involving the heart and brain. The link between elevated serum glucose and end organ damage is well established. ${ }^{14}$ The mechanisms through which hyperglycemia mediates vascular damage are complex and multifactorial, including formation of advanced glycation end products and increased release of proinflammatory molecules, as well as impaired endothelial, fibrinolytic, and platelet function. ${ }^{15}$

Numerous studies have demonstrated a link between glycemic control and reductions in microvascular damage. In type I diabetics, there is conclusive evidence from the Diabetes Control and Complications Trial (DCCC) that intensive glucose control reduces microvascular complications such as retinopathy, nephropathy, and neuropathy. ${ }^{16}$ No differences were seen in macrovascular complications during the initial trial. However, for the $93 \%$ of trial participants who were then followed for a mean of 17 years in the Epidemiology of Diabetes Interventions and Complications (EPIC) Study, intensive glucose control was associated with a reduction in CV events (RR, $42 \%$; 95\% CI, 9\%-63\%; P = .02). ${ }^{17}$ These findings support the hypothesis that longer treatment durations may be needed to significantly affect CV endpoints.

An observational study in type 2 diabetes, the United Kingdom Prospective Diabetes Study UKPDS, demonstrated the association between hyperglycemia and both microvascular and macrovascular complications. ${ }^{18}$ This study enrolled 4,209 diabetic individuals soon after their diagnosis and followed them for a median of 10 years. Various drugs including metformin, sulfonylureas, and insulin were utilized. Each $1 \%$ reduction in $\mathrm{A} 1 \mathrm{C}$ was associated with a $21 \%$ risk reduction for any endpoint related to diabetes (95\% CI, $17 \%-24 \% ; P<.0001$ ), a $21 \%$ reduction for deaths related to diabetes $(95 \% \mathrm{CI}$, $15 \%-27 \% ; P<.0001)$, and a $14 \%$ reduction for myocardial infarction $(95 \% \mathrm{CI}, 8 \%-21 \% ; P<.0001)$. The risk reduction in microvascular complications was even greater (RR, 37\%; 95\% CI, 33\%-41\%; $P<.0001)$.

In an effort to more fully determine the effectiveness of intensive glucose lowering on macrovascular complications, 3 clinical trials have been conducted. ${ }^{19-21}$ The Action to Control Cardiovascular Risk in Diabetes (ACCORD) was a randomized trial of 10,251 patients with a median A1C of $8.1 \% .{ }^{19}$ Patients were assigned to intensive therapy with a target A1C of less than $6.0 \%$ or standard therapy with a target $\mathrm{A} 1 \mathrm{C}$ of $7.0 \%$ to $7.9 \%$. Treatments included various combinations of insulin, metformin, sulfonylureas, and thiazolinediones. At 1 year, stable median A1C levels of $6.4 \%$ and $7.5 \%$ were achieved in the intensive and standard therapy groups, respectively. After a mean follow-up of 3.5 years, the primary outcome, a composite of nonfatal myocardial infarction, nonfatal stroke, or death from CV causes, was not significantly different between groups (HR, 0.90; 95\% CI, 0.78-1.04; $P=.16)$. Particularly concerning was the finding that mortality was higher in the intensive therapy group (HR, 1.22; 95\% CI, 1.01-1.46; $P=.04$ ), leading to early termination of the trial.

A second trial, Action in Diabetes and Vascular Disease: Preterax and Diamicron Modified Release Controlled Evaluation (ADVANCE), was a randomized, controlled, multicenter trial of 11,140 patients with type 2 diabetes that compared standard glucose control to intensive glucose control (defined as a A1C of $6.5 \%$ or less)..$^{20}$ The intensive control group received gliclazide, a sulfonylurea not available in the United States, plus additional therapy as directed by their physician. The primary endpoint was a composite of macrovascular and microvascular events. After a median follow-up of 5 years, the mean A1C in the intensive control group was $6.5 \%$ compared to $7.3 \%$ in the standard control group. The combined incidence of macrovascular and microvascular events was $18.1 \%$ in the intensive control group 
versus $20.0 \%$ in the standard control group (HR, $0.90 ; 95 \%$ CI, 0.82-0.98; $P=.01)$. This risk reduction, however, was due primarily to a $21 \%$ decrease in new or worsening nephropathy. When analyzed separately, intensive control was associated with a reduced risk of microvascular events (HR, 0.86; 95\% CI, $0.77-0.97, P=.01$ ) but no difference in the risk of macrovascular events $(\mathrm{HR}, 0.94 ; 95 \% \mathrm{CI}$, $0.84-1.06 ; P=.32$ ).

The third trial, the Veterans Affairs Diabetes Trial (VADT), randomized 1,791 military veterans with type 2 diabetes and a mean baseline A1C of $9.4 \% .{ }^{21}$ The mean age of participants was 60.4 years, and the mean number of years since the diagnosis of diabetes was 11.5. Patients were randomized to either intensive therapy with a goal of reducing the A $1 \mathrm{C}$ by $1.5 \%$ or standard therapy. The primary outcome was the first occurrence of any composite of CV events. The median A1C stabilized after 6 months at $6.9 \%$ in the intensive therapy group versus $8.4 \%$ in the standard therapy group. However, after a median follow-up of 5.6 years, there was no reduction in the primary endpoint $(\mathrm{OR}, 0.88$; 95\% CI, 0.74-1.05; $P=.14$ ).

Along with the findings from these 3 trials, SAVOR-TIMI 53 and EXAMINE raise further questions about the utility of glycemic control as a surrogate for macrovascular damage. Moreover, the trial findings suggest that the duration of diabetes, baseline presence of CV risk factors or disease, and length of follow-up are all important factors that must be taken into account when assessing a drug's effectiveness for preventing CV complications.

Evidence from ACCORD, ADVANCE, and VADT suggest that patients with more advanced diabetes and a higher prevalence of prior macrovascular disease do not derive benefit from intensive glucose control at least over 5 years of follow-up and that mortality may potentially be increased. Hypoglycemia that occurred frequently in ACCORD and VADT was a risk factor for CV complications. Conversely, based on the findings from UKPDS, newly diagnosed diabetics and those without prior CVD may derive greater long-term benefit from intensive glycemic control, assuming hypoglycemia can be minimized. These factors are reflected in the most recent guidelines from the American Diabetes Association that recommend a target $\mathrm{A} 1 \mathrm{C}$ for most patients of less than $7 \%$ but note a more stringent goal of less than $6.5 \%$ may be appropriate for newly diagnosed individuals with no history of microvascular or macrovascular disease and a more lenient goal of less than $8 \%$ may be more appropriate for those with advanced disease and more complications. ${ }^{22}$

Lastly, it should be kept in mind that reducing hyperglycemia is only one factor that must be considered in reducing a patient's overall risk for $\mathrm{CV}$ disease or stroke. This was demonstrated in the Steno-2 study that involved long-term interventions targeted to reduce $\mathrm{A} 1 \mathrm{C}$, blood pressure, cholesterol, triglycerides, and urinary albumin excretion based on national guidelines. ${ }^{23}$ The $\mathrm{A} 1 \mathrm{C}$ goal was less than $6.5 \%$ using diet, exercise, and various older drugs including metformin, sulfonylureas, and insulin as needed. The study randomized 160 patients who had mean age 55.1 years with type 2 diabetes and microalbuminuria. After a mean follow-up of 7.8 years, significant reductions were seen in both macrovascular and microvascular endpoints: CVD (HR, 0.47; 95\% CI, 0.24-0.73), nephropathy (HR, 0.39; 95\% CI, $0.17-0.87$ ), retinopathy (HR, 0.42; 95\% CI, 0.210.86 ), and autonomic neuropathy (HR, 0.37; 95\% CI, 0.18-0.79). This study provides evidence that a comprehensive program targeting lifestyle changes and risk factor modification will likely be the most effective approach for preventing long-term complications.

\section{Conclusions}

Diabetes is a rapidly escalating public health problem. In 2012, the total costs of diagnosed diabetes were estimated at $\$ 245$ billion. ${ }^{24}$ CVD is a major contributor to morbidity and mortality in patients with type 2 diabetes. Additional clinical trials assessing the CV impact of newer drugs such as the DPP-4 inhibitors are ongoing and should be completed by 2017. However, the evidence to date for the DPP-4 inhibitors as well as other antihyperglycemic drugs has been disappointing. For patients with no prior history of CV disease, intensive glucose-lowering therapy soon after diagnosis may be effective, if frequent hypoglycemia can be avoided. For patients with more advanced disease and multiple comorbidities, no single intervention is enough. Rather, a multifaceted strategy should be implemented that targets blood glucose, blood pressure, serum lipids, and other CV risk factors. Less stringent glycemic goals may be appropriate for many of these patients. Frequent or severe hypoglycemia is an indication that treatment regimens should be modified. Although the lack of CV benefit in the recent DPP-4 inhibitor trials was disappointing, the absence of adverse effects on vascular disease is reassuring and the possibility of benefit with longer duration follow-up has not been fully explored. Caution is advised, however, for 
patients at risk or with a history of heart failure until this issue can be further assessed. Overall, given the relatively high costs of the DPP-4 inhibitors, the lack of CV benefit will likely keep these agents as add-on therapy. Pharmacists should bear in mind that many individuals with diabetes remain undiagnosed until complications appear. Thus ongoing efforts for early detection and education are also needed.

\section{REFERENCES}

1. National Diabetes Statistics, 2011. National Diabetes Information Clearinghouse (NDIC). US Department of Health and Human Services. http://diabetes.niddk.nih.gov/dm/pubs/ statistics/\#hds. Accessed May 8, 2014.

2. Selvin E, Paminello CM, Sacks DB, Coresh J. Trends in prevalence and control of diabetes in the United States 19881994 and 1999-2010. Ann Intern Med. 2014;160:517-525.

3. Loke YK, Kwok CS, Singh S. Comparative cardiovascular effects of thiazolidinediones; systematic review and metaanalysis of observational studies. BMJ. 2011;342:d1309.

4. Choy-Shan A, Zinn A, Shah B, et al. Effect of rosiglitazone on survival in patients with diabetes mellitus treated for coronary artery disease. Coronary Artery Dis. 2012;23:354e358.

5. FDA lifts rosiglitazone prescribing restrictions. Medscape. November 25, 2013.

6. Guidance for industry: Diabetes mellitus- evaluating cardiovascular risk in new antidiabetic therapies to treat type 2 diabetes. US Food and Drug Administration. December 2008. www.fda.gov/downloads/Drugs/GuidanceComplianceRegulatoryInformation/Guidances/ucm071627.pdf. Accessed May 8, 2014.

7. Kwok AJ, Mashar M, Hlavandi, Sabir I. DPP-IV inhibitors: Beyond glycemic control? Trend Cardiovasc Med. 2014;24:157-164.

8. Monami M, Ahrén B, Dicembrini I, Mannucci E. Dipeptidyl peptidase-4 inhibitors and cardiovascular risk: A meta-analysis of randomized clinical trials. Diabetes Obes Metab. 2013;15:112-120.

9. Frederich R, Alexander JH, Fiedorek FT, et al. A systematic assessment of cardiovascular outcomes in the saxagliptin drug development program for type 2 diabetes. Postgrad Med. 2010;122:16-27.

10. Scirica BM, Bhatt DL, Braunwald E, et al. Saxagliptin and cardiovascular outcomes in patients with type 2 diabetes mellitus. N Eng J Med. 2013;369:1317-1326.

11. White WB, Pratley R, Fleck P, et al. Cardiovascular safety of the dipeptidyl peptidase-4 inhibitor alogliptin in type 2 diabetes mellitus. Diabetes Obes Metab. 2013;15:668-673
12. Monami M, Dicembrini I, Mannucci E. Dipeptidyl peptidase-4 inhibitors and heart failure: A meta-analysis of randomized clinical trials [published online ahead of print March 5, 2014]. Nutr Metab Cardiovasc Dis.

13. FDA Drug Safety Communication: FDA to review heart failure risk with diabetes drug saxagliptin (marketed as Onglyza and Kombiglyze XR). Issued February 11, 2014. http://www.fda.gov/drugs/drugsafety/ucm 385287.htm. Accessed June 4, 2014.

14. Terry T, Raravikar K, Chokrungvaronon N, et al. Does aggressive glycemic control benefit macrovascular and microvascular disease in type 2 diabetes?: Insights from ACCORD, ADVANCE and VADT. Curr Cardiol Rep. 2012;14:79-88.

15. Singh A, Donnino R, Weintraub H, et al. Effect of strict glycemic control in patients with diabetes mellitus on frequency of macrovascular events. Am J Cardiol. 2013;112:1033-1038.

16. The Diabetes Control and Complications Trial Research Group. The effect of intensive treatment of diabetes on the development and progression of longterm complications in insulin-dependent diabetes mellitus. N Engl J Med. 1993;329: 977-986.

17. Nathan DM, Cleary PA, Backlund JY, et al; for the Diabetes Control and Complications Trial/Epidemiology of Diabetes Interventions and Complications (DCCT/EDIC) Study Research Group. Intensive diabetes treatment and cardiovascular disease in patients with type 1 diabetes. $N$ Engl J Med. 2005;353:2643-2653.

18. Stratton IM, Adler AI, Matthews DR, et al. Association of glycaemia with macrovascular and microvascular complications of type 2 diabetes (UKPDS 35): Prospective observational study. BMJ. 2000;321:405-412.

19. The Action to Control Cardiovascular Risk in Diabetes (ACCORD) Study Group. Effects of intensive glucose lowering in type 2 diabetes. N Engl J Med. 2008;358:2545-2559.

20. The ADVANCE Collaborative Group. Intensive blood glucose control and vascular outcomes in patients with type 2 diabetes. N Engl J Med. 2008;358:2560-2572.

21. Duckworth W, Abraira C, Moritz T, et al. Glucose control and vascular complications in veterans with type 2 diabetes. N Engl J Med. 2009;360:129-139.

22. Standards of Medical Care in Diabetes - 2014. American Diabetes Association. Diabetes Care. 2014;37(Suppl 1): S14-S80.

23. Gaede P, Vedel P, Larsen N, et al. Multifactorial intervention and cardiovascular disease in patients with type 2 diabetes. N Engl J Med. 2003;348:383-393.

24. American Diabetes Association. Economic costs of diabetes in the U.S. in 2012. Diabetes Care. 2013;36(4): 1033-1046. 